\title{
EGFR variant allele frequency predicts EGFR-TKI efficacy in lung adenocarcinoma: a multicenter study
}

\author{
Balazs Gieszer ${ }^{1 \#}$, Zsolt Megyesfalvi ${ }^{1,2,3 \#}$, Viktoria Dulai ${ }^{2}$, Judit Papay ${ }^{4}$, Ilona Kovalszky ${ }^{4}$, Jozsef Timar ${ }^{5}$, \\ Janos Fillinger ${ }^{1,2}$, Tunde Harko ${ }^{2}$, Orsolya Pipek ${ }^{6}$, Vanda Teglasi ${ }^{4}$, Eszter Regos ${ }^{4}$, Gergo Papp ${ }^{4}$, \\ Zoltan Szallasi $^{7,8}$, Viktoria Laszlo ${ }^{2,3}$, Ferenc Renyi-Vamos ${ }^{1,2}$, Gabriella Galffy ${ }^{9}$, Csaba Bodor ${ }^{4}$, \\ Balazs Dome ${ }^{1,2,3 *}$, Judit Moldvay ${ }^{2,7 *}$
}

${ }^{1}$ Department of Thoracic Surgery, Semmelweis University and National Institute of Oncology, Budapest, Hungary; ${ }^{2}$ National Koranyi Institute of Pulmonology, Budapest, Hungary; ${ }^{3}$ Department of Thoracic Surgery, Comprehensive Cancer Center, Medical University of Vienna, Vienna, Austria; ${ }^{4} 1$ st Department of Pathology and Experimental Cancer Research, Semmelweis University, Budapest, Hungary; ${ }^{5}$ 2nd Department of Pathology, Semmelweis University, Budapest, Hungary; ${ }^{6}$ Department of Physics of Complex Systems, Eotvos Lorand University, Budapest, Hungary; ${ }^{7}$ MTA-SE NAP, Brain Metastasis Research Group, Hungarian Academy of Sciences, Budapest, Hungary; ${ }^{8}$ Computational Health Informatics Program, Boston Children's Hospital, Harvard Medical School, Boston, MA, USA; ${ }^{9}$ Torokbalint County Institute of Pulmonology, Torokbalint, Hungary

Contributions: (I) Conception and design: B Gieszer, Z Megyesfalvi, B Dome, J Moldvay; (II) Administrative support: V Dulai, J Papay, I Kovalszky, J Timar, J Fillinger, T Harko, G Papp, Z Szallasi, F Renyi-Vamos, G Galffy, C Bodor; (III) Provision of study materials or patients: J Papay, I Kovalszky, J Timar, J Fillinger, T Harko, V Teglasi, E Regos, G Papp, F Renyi-Vamos, G Galffy, C Bodor; (IV) Collection and assembly of data: B Gieszer, Z Megyesfalvi, V Dulai; (V) Data analysis and interpretation: B Gieszer, Z Megyesfalvi, O Pipek, V Laszlo, B Dome, J Moldvay; (VI) Manuscript writing: All authors; (VII) Final approval of manuscript: All authors.

\#These authors contributed equally to this work.

*These authors contributed equally for the senior authorship.

Correspondence to: Balazs Dome, MD, PhD. Department of Thoracic Surgery, Comprehensive Cancer Center, Medical University of Vienna, Waehringer Guertel 18-20, A-1090 Vienna, Austria. Email: balazs.dome@meduniwien.ac.at; Zsolt Megyesfalvi, MD. Department of Thoracic Surgery, National Institute of Oncology-Semmelweis University, Rath Gyorgy u. 7-9, 1122 Budapest, Hungary. Email: megyesfalvi.zsolt@semmelweis-univ.hu.

Background: Although lung adenocarcinoma (LADC) with sensitizing mutations of the epidermal growth factor receptor (EGFR) is highly sensitive to EGFR tyrosine kinase inhibitors (EGFR-TKIs), in most cases disease progression inevitably occurs. Our aim was to investigate the predictive and prognostic significance of adjusted tumoral EGFR variant allele frequency (EGFR-aVAF) in the above setting.

Methods: Eighty-nine Caucasian advanced-stage LADC patients with known exon-specific EGFR mutations undergoing EGFR-TKI treatment were included. The correlations of EGFR-aVAF with clinicopathological variables including progression-free and overall survival (PFS and OS, respectively) were retrospectively analyzed.

Results: Of 89 EGFR-mutant LADC patients, 46 (51.7\%) had exon 19 deletion, while 41 (46.1\%) and 2 (2.2\%) patients had exon 21- and exon 18-point mutations, respectively. Tumoral EGFR-aVAF was significantly higher in patients harboring EGFR exon 19 mutations than in those with exon 21-mutant tumors $(\mathrm{P}<0.001)$. Notably, patients with $E G F R$ exon 19 mutant tumors demonstrated significantly improved PFS $(\mathrm{P}=0.003)$ and $\mathrm{OS}(\mathrm{P}=0.02)$ compared to patients with exon 21 mutations. Irrespective of specific exon mutations, a statistically significant positive linear correlation was found between EGFR-aVAF of tumoral tissue and PFS $(\mathrm{r}=0.319 ; \mathrm{P}=0.002)$. High $(\geq 70 \%)$ EGFR-aVAF was an independent predictor of longer PFS [vs. low (<70\%) EGFR-aVAF; median PFSs were 52 vs. 26 weeks, respectively; $\mathrm{P}<0.001]$. Additionally, patients with high EGFR-aVAF also had significantly improved OS than those with low EGFR-aVAF $(\mathrm{P}=0.011)$.

Conclusions: Our study suggests that high $(\geq 70 \%)$ EGFR-aVAF of tumoral tissue predicts benefit from 
EGFR-TKI treatment in advanced LADC and, moreover, that exon 19 EGFR mutation is associated with high EGFR-aVAF and improved survival outcomes.

Keywords: Epidermal growth factor receptor mutation (EGFR mutation); lung adenocarcinoma (LADC); variant allele frequency (VAF)

Submitted Jul 06, 2020. Accepted for publication Nov 25, 2020.

doi: $10.21037 /$ tlcr-20-814

View this article at: http://dx.doi.org/10.21037/tlcr-20-814

\section{Introduction}

Lung cancer is the most frequently diagnosed malignancy worldwide (11.6\% of the total cases) and the leading cause of cancer-related mortality $(18.4 \%$ of the total cancer deaths) (1). Histologically, non-small cell lung cancer (NSCLC) is the predominant lung cancer subtype and more than $40 \%$ of all NSCLCs diagnosed are lung adenocarcinomas (LADCs) (1). However, not all LADCs are the same, and inter-tumoral heterogeneity exists both in terms of pathological and molecular features (2).

Epidermal growth factor receptor $(E G F R)$ mutations are the second most common oncogenic driver events in LADC, accounting for approximately $15 \%$ of all LADCs in Caucasian patients and about $40 \%$ to $50 \%$ in Asian patients $(3,4)$. EGFR is a member of the ErbB family of tyrosine kinase receptors that is expressed in some normal epithelial, mesenchymal, and neurogenic tissue with cytoplasmic kinase activity transducing important growth factor signaling $(5,6)$. However, in malignant tumors including LADC, EGFR is often constantly stimulated due to the sustained production in the tumor microenvironment of EGFR ligands, or as a result of a mutation in EGFR itself that locks the receptor in a state of continuous activation $(7,8)$. About $90 \%$ of activating EGFR mutations are short in-frame deletions in exon 19 or point mutations in exon 21 often referred to as "classical" EGFR mutations $(9,10)$. Exon 18 mutations are rare and relatively homogenous (compared to other rare mutations such as EGFR exon 20 insertions) as they represent about $4 \%$ of all EGFR mutations $(9,10)$. Importantly, in LADC, these EGFR-sensitizing mutations confer sensitivity both to first-, second- and thirdgeneration EGFR tyrosine kinase inhibitors (EGFRTKIs) such as gefitinib, erlotinib, dacomitinib, afatinib and osimertinib in patients with advanced-stage disease (11-13).

Over the past decade, the application of EGFRTKIs have led to a new era in the treatment of LADC. Accordingly, EGFR-TKIs improve both the progression- free survival (PFS) [10.8 vs. 5.4 months in the chemotherapy $(\mathrm{CHT})$ group; $\mathrm{P}<0.001]$ and overall survival (OS) $(30.5$ vs. 23.6 months in the CHT group; $\mathrm{P}=0.31$ ) in patients who were selected on the basis of EGFR-sensitizing mutations (14). Still, the objective response rate to EGFRTKIs in patients carrying EGFR-sensitizing mutations is only $70 \%$ to $80 \%$, and while some patients show clear survival benefit to TKIs others failed to respond properly $(15,16)$. Therefore, in order to assess the effectiveness of current treatment options, it is crucial to understand the intrinsic and extrinsic factors that influence the responsiveness to TKIs in these patients.

Sensitivity to EGFR-TKIs is associated with female sex, never-smoking status and Asian ethnicity, however, such clinical factors are in fact predictors of EGFR mutations rather than true treatment-related prognosticators for TKI efficacy $(14,15,17,18)$. Nevertheless, different $E G F R$ mutation subtypes and molecular characteristics can also determine different predictive and prognostic features (15). In addition, differences in the proportion of tumor cells (TCs) harboring EGFR mutations might also contribute to therapy response, since only a fraction of cancer cells in an individual patient carry heterozygous activating mutations, whereas other cancer cells carry wild-type EGFR (19-22). Accordingly, previous studies on Asian patients suggest that higher relative $E G F R$ mutational abundance might predict benefit from EGFR-TKI treatment $(19,23,24)$.

Presently, the biological and clinical relevance of adjusted tumoral EGFR variant allele frequency (EGFR-aVAF) in terms of prognosis and clinical response to EGFR-TKIs is still mostly unclear. Therefore, in order to improve patient selection and to better understand the influence of EGFRaVAF in this setting with regards to therapeutic approaches, our aim was to assess the relationship between EGFR-aVAF and response to EGFR-TKIs in a homogenous patient cohort of Caucasian LADC patients.

We present the following article in accordance with the 
STROBE reporting checklist (available at http://dx.doi. org/10.21037/tlcr-20-814).

\section{Methods}

\section{Ethics statement}

The present study was directed in accordance with the guidelines of the Helsinki Declaration (revised in 2013) of the World Medical Association. The study was approved by the national level ethics committee (Hungarian Scientific and Research Ethics Committee of the Medical Research Council, ETT-TUKEB, 7214-1/2016/EKU). The need for individual informed consent for this retrospective study was waived. After clinical information was collected, patient identifiers were removed, and subsequently, patients cannot be identified either directly or indirectly.

\section{Study population}

Based on our inclusion/exclusion criteria, 89 pathologically confirmed advanced-stage LADC patients were included in this multi-center, retrospective study, who received EGFR-TKI therapy mainly in the following two Hungarian medical centers between 2008 and 2020: Torokbalint County Institute of Pulmonology, Torokbalint; and Department of Pulmonology of the Semmelweis University, Budapest, Hungary. Of note, all ten participating medical centers are enlisted in Table S1. All tumor tissues were tested for EGFR mutations required for anti-EGFR therapy and all samples were retrieved from treatmentnaïve patients. Based on our inclusion criteria, cytologically or histologically verified unresectable stage IIIb or stage IV patients were included who received either gefitinib or erlotinib as first- or second-line treatment. According to the therapy guidelines of the host institutes, only patients with Eastern Cooperative Oncology Group (ECOG) performance status (PS) $0-1$ were included. With regards to our exclusion criteria, patients with concomitant mutations in two or more exons, or patients harboring resistance mutations such as T790M substitution in exon 20 were excluded. Additionally, all cases where the exact percentage of neoplastic cells was not available were also subsequently excluded. Patients treated with afatinib were excluded due to the relatively small number of cases. Similarly, patients in whom TKI therapy was suspended by the reason of drug-related toxicities like hepato- and cardiotoxicities, or patients treated with EGFR-TKIs as third-line therapy were also excluded. Finally, patients who received EGFRTKI therapy for a period less than 4 weeks or the cause of death was not related to lung cancer progression were also excluded. Clinicopathological data regarding gender, age at lung cancer diagnosis, smoking history, type of EGFR exon mutation, EGFR-aVAF, treatment and survival data for the included patients were retrospectively collected from medical records and/or records from the National Health Insurance Office or Central Statistical Office.

\section{Treatment}

Diagnostic and therapeutic approaches were conducted in accordance with the individual institutional guidelines and with the current National Comprehensive Cancer Network (NCCN) guidelines with no differences across the host institutes (25). All included patients received either gefitinib or erlotinib as first- or second-line systemic therapy on a daily basis (250 and $150 \mathrm{mg}$, respectively) until disease progression. In case of patients who received first-line CHT before the initiation of TKI therapy, patients were treated with platinum-based standard of care CHT regimens. According to the national treatment financing scheme, all EGFR-TKI-treated patients had to return to the hospital every month for chest X-ray and clinical check-up, and the clinical response to treatment was classified based on follow-up CT scans every 3 months by using the response evaluation criteria in solid tumors (RECIST 1.0) (26).

\section{EGFR mutation analysis}

Tissue samples were acquired during diagnostic procedures including wedge resection surgery and bronchoscopic- or transthoracic needle biopsy. The diagnosis of LADC of each case was confirmed on a freshly prepared hematoxylin and eosin stained slide. As this estimate is critical for the study, the exact proportion of neoplastic cells was reassessed by two independent expert histopathologists. All mutational analyses were performed at the $1^{\text {st }}$ Department of Pathology and Experimental Cancer Research of the Semmelweis University. Genomic DNA was extracted using the High Pure PCR Template Preparation Kit (Roche, Basel, Switzerland) in accordance with the manufacturer's instructions, and all samples underwent testing for mutation in EGFR codon (in exon 18, 19, 21) using the Therascreen EGFR Pyro Kit (Qiagen, Germany) on a PyroMark ${ }^{\mathrm{TM}}$ Q24 (Qiagen) pyrosequencing instrument. The percentage of 
Table 1 Patient characteristics and adjusted tumoral EGFR-VAF in human LADC

\begin{tabular}{|c|c|c|c|}
\hline Characteristics & $\begin{array}{l}\text { Number of } \\
\text { patients (\%) }\end{array}$ & $\begin{array}{c}\text { Mean EGFR- } \\
\text { aVAF, } \%\end{array}$ & $P$ value ${ }^{a}$ \\
\hline All patients & 89 (100.0) & & \\
\hline Age (years) & & & $0.93^{\mathrm{b}}$ \\
\hline$<65$ & $36(40.4)$ & 63.53 & \\
\hline$\geq 65$ & $53(59.6)$ & 64.6 & \\
\hline Gender & & & $0.809^{b}$ \\
\hline Male & $25(28.1)$ & 64.12 & \\
\hline Female & 64 (71.9) & 64.19 & \\
\hline Smoking history & & & $0.467^{\mathrm{c}}$ \\
\hline Never smoker & $48(51.7)$ & 64.46 & \\
\hline Ex-smoker & $10(11.2)$ & 73.3 & \\
\hline Current smoker & $14(15.7)$ & 58.5 & \\
\hline No data & $19(21.3)$ & - & \\
\hline Therapeutic agent & & & $0.428^{b}$ \\
\hline Gefitinib & $58(65.2)$ & 61.64 & \\
\hline Erlotinib & $31(34.8)$ & 68.9 & \\
\hline Treatment line & & & $0.882^{b}$ \\
\hline First-line & $46(51.7)$ & 63.35 & \\
\hline Second-line & $43(48.3)$ & 65.05 & \\
\hline \multicolumn{4}{|c|}{ EGFR exon mutation } \\
\hline Exon 18 & $2(2.2)$ & - & $<0.001^{\mathrm{b}}$ \\
\hline Exon 19 & $46(51.7)$ & 75.04 & \\
\hline Exon 21 & $41(46.1)$ & 51.44 & \\
\hline
\end{tabular}

a, $\mathrm{P}$ values refer to mean EGFR-aVAF between patient subgroups; ${ }^{b}$, Mann-Whitney U test; ${ }^{c}$, Kruskal-Wallis test; ${ }^{d}$, not included in the statistical calculation. EGFR, epidermal growth factor receptor; EGFR-aVAF, adjusted EGFR variant allele frequency; LADC, lung adenocarcinoma.

mutated nucleic acid was calculated with the equipment software (Qiagen PyroMark ${ }^{\mathrm{TM}}$ ), relating the peak of mutated base to that of the wild-type base, which was considered $100 \%$. The obtained VAF for each patient was then normalized to the proportion of neoplastic cells in each specimen using the following formula:

$$
\text { Adjusted VAF }(\mathrm{aVAF})=\frac{\mathrm{VAF}}{\mathrm{TC} \%} \times 100
$$

Where VAF represents the percentage of the EGFR variant alleles determined by the pyrosequencing assay and TC\% is the estimated percentage of neoplastic cells.

\section{Statistical analyses}

All statistical analyses were performed using the SPSS Statistics 23.0 package (SPSS Inc., Chicago, IL, USA). Data distribution was verified by the KolmogorovSmirnov normality test. EGFR-aVAF of tumoral tissue as a continuous variable was analyzed with regards to dichotomized clinicopathological variables by MannWhitney $U$ test and Kruskal-Wallis test. The co-primary endpoints were PFS and OS. PFS was defined as the time from commencement of gefitinib or erlotinib treatment to disease progression according to the aforementioned RECIST 1.0 criteria. OS was defined as the interval between the initiation of medication and death related to progressive disease. Clinical follow-up was closed on the $1^{\text {st }}$ of April, 2020. Survival curves were estimated by KaplanMeier plots and the differences between different groups were compared using the log-rank test. The association between EGFR-aVAF as continuous variable and PFS and OS was also evaluated by using the Spearman's correlation coefficient. The value of linear correlation coefficient $(\mathrm{r})$ varies from -1 to 1 both values inclusive. No linear correlation $(\mathrm{r}=0)$, weak positive correlation $(0<\mathrm{r} \leq 0.3)$, moderate positive linear correlation $(0.3<\mathrm{r} \leq 0.7)$, strong positive linear correlation $(0.7<\mathrm{r} \leq 1)(27)$. The independent prognostic value of the clinicopathological variables was studied with Cox proportional hazard regression model, which was adjusted for EGFR-aVAF and age (as continuous variables), gender (male versus female), EGFR exon mutation (exon 19 versus exon 21), therapeutic agents (gefitinib versus erlotinib) and treatment line (first- versus second-line). All reported $\mathrm{P}$ values are two-sided, and a level of 0.05 or less was considered statistically significant.

\section{Results}

\section{Patient characteristics and EGFR-aVAF}

After applying the exclusion criteria, 89 LADC patients with known EGFR gene mutations were enrolled in the study whose clinicopathological characteristics are summarized in Table 1. All patients had advanced-stage disease and Caucasian background. Median age of all cases was 67 (range, 34-92) years and patients were predominantly female (71.9\%). A total of 46 (51.7\%) patients had exon 19 deletion, while $41(46.1 \%)$ and $2(2.2 \%)$ patients had exon 
21- and exon 18-point mutations, respectively. Median age was 61,66 and 70 years in exon mutation subgroups 18, 19 and 21 , respectively (with no significant differences in age distribution, $\mathrm{P}=0.332$; data not shown). As for therapeutic approaches, 58 (65.2\%) patients received gefitinib, while 31 $(34.8 \%)$ patients were treated with erlotinib.

In order to study the clinical relevance of mutational percentage of tumoral tissue, we performed comparative statistical analyses of EGFR-aVAF and clinicopathological variables. Out of all 89 cases, 72 cases showed EGFRaVAF between $5 \%$ and $94 \%$ and 17 patients exhibited EGFR-aVAF $\geq 95 \%$ (Figure $1 A$ ). In case of six patients the EGFR-aVAF of tumoral tissue was $<20 \%$. Interestingly, the adjusted VAF was significantly higher in patients harboring EGFR exon 19 mutations than those with exon 21 mutant tumors $(\mathrm{P}<0.001$; Table 1, Figure $1 B)$. There were no statistically significant differences in the mean EGFR-aVAF with respect to age $(\mathrm{P}=0.93)$, gender $(\mathrm{P}=0.809)$, or smoking history $(\mathrm{P}=0.467)$.

\section{EGFR exon 19 mutation associates with superior survival outcomes}

The median PFS and OS of the full cohort was 38 and 72 weeks, respectively. At the time of the closing date of the clinical follow-up, all patients with EGFR exon 18 mutations, 42 patients with exon 19 mutations and 39 patients with exon 21 mutations had experienced disease progression after EGFR-TKI therapy. Due to the small number of patients in EGFR exon 18-mutated subgroup, statistical analyses were performed solely by comparing the median PFS and OS of exon subgroups 19 and 21 . Accordingly, as shown in Figure 2A, LADC patients with tumors harboring EGFR exon 19 mutations had significantly improved median PFS than those with exon 21 mutations (median PFSs were 44 vs. 25 weeks, respectively; $\mathrm{P}=0.003$ ). In line with the PFS data, EGFR exon 19 mutations were significantly associated with longer OS as well (vs. exon 21 mutation, median OSs were 76 vs. 57 weeks, respectively; $\mathrm{P}=0.02$; Figure $2 \mathrm{~B}$ ). With regards to the administered therapeutic agents, no significant differences have been observed neither in PFS ( $\mathrm{P}=0.654$; Figure $2 C)$ nor in OS $(\mathrm{P}=0.665$; Figure $2 D)$ in patients treated with gefitinib vs. erlotinib. Of note, the treatment line of EGFR-TKI did not influence the survival outcomes neither (Figure S1A,B). As for smoking history, there was no significant difference in PFS between never-smoker versus ever-smoker patients $(\mathrm{P}=0.099$; Figure S1C). Interestingly, however, Kaplan-
Meyer curves demonstrated significantly longer median OS in never-smoker patients (vs. ever-smokers, median OSs were 106 vs. 52 weeks, respectively, $\mathrm{P}=0.007$; Figure S1D).

\section{EGFR-aVAF bas clinical utility in predicting survival outcomes in LADC patients treated with EGFR-TKIs}

Next, we evaluated the survival outcomes of TKItreated $E G F R$-mutant LADC patients with regards to adjusted tumoral variant allele frequencies. Importantly, a statistically significant positive linear correlation was found between EGFR-aVAF and PFS ( $\mathrm{r}=0.319 ; \mathrm{P}=0.002$, Spearman's correlation; Figure $3 A$ ). In contrast, no significant correlation was found between EGFR-VAF and OS, although the correlation coefficient was found to be clinically notable $(r=0.208 ; \mathrm{P}=0.061$, Spearman's correlation; Figure $3 B$ ). In order to rule out the potential confounding effects of Spearman's correlation and to evaluate the survival outcomes with Kaplan-Meier methods, patients were categorized by the median EGFR-aVAF (70\%) of tumoral tissue. Therefore, we grouped patients into low $(<70 \%)$ and high ( $\geq 70 \%)$ EGFR-aVAF categories and found that patients with high adjusted tumoral EGFR-VAF had significantly longer PFS than those in the low EGFR-aVAF group (median PFSs were 52 vs. 26 weeks, respectively; $\mathrm{P}<0.001$, Figure $3 C$ ). Additionally, patients with high EGFR-aVAF also had significantly improved OS (vs. those with low EGFR-aVAF; median OSs were 94 vs. 57 weeks, respectively; $\mathrm{P}=0.011$, Figure $3 D$ ).

In order to assess if the predictive value of tumoral EGFRaVAF was independent from other clinicopathological factors, we performed a multivariate Cox regression analysis (Table 2). The model was adjusted for clinicopathological variables such as EGFR-aVAF, age, gender, EGFR exon mutation, therapeutic agents and treatment line. Importantly, we found that EGFR-aVAF of tumoral tissue remained a significant prognostic factor for PFS [continuous variable, hazard ratio (HR): $-0.009,95 \%$ confidence interval (CI): 0.982-0.999; $\mathrm{P}=0.042$; Table 2]. Besides, Cox regression analysis revealed that the specific exon mutations (nominal variable, HR: 0.284, 95\% CI: 1.017-1.735; $\mathrm{P}=0.037)$ also influence the PFS independently.

\section{Discussion}

In the era of precision and individualized cancer therapy precise definition of tumor type including comprehensive histological classification, and description of clinically 


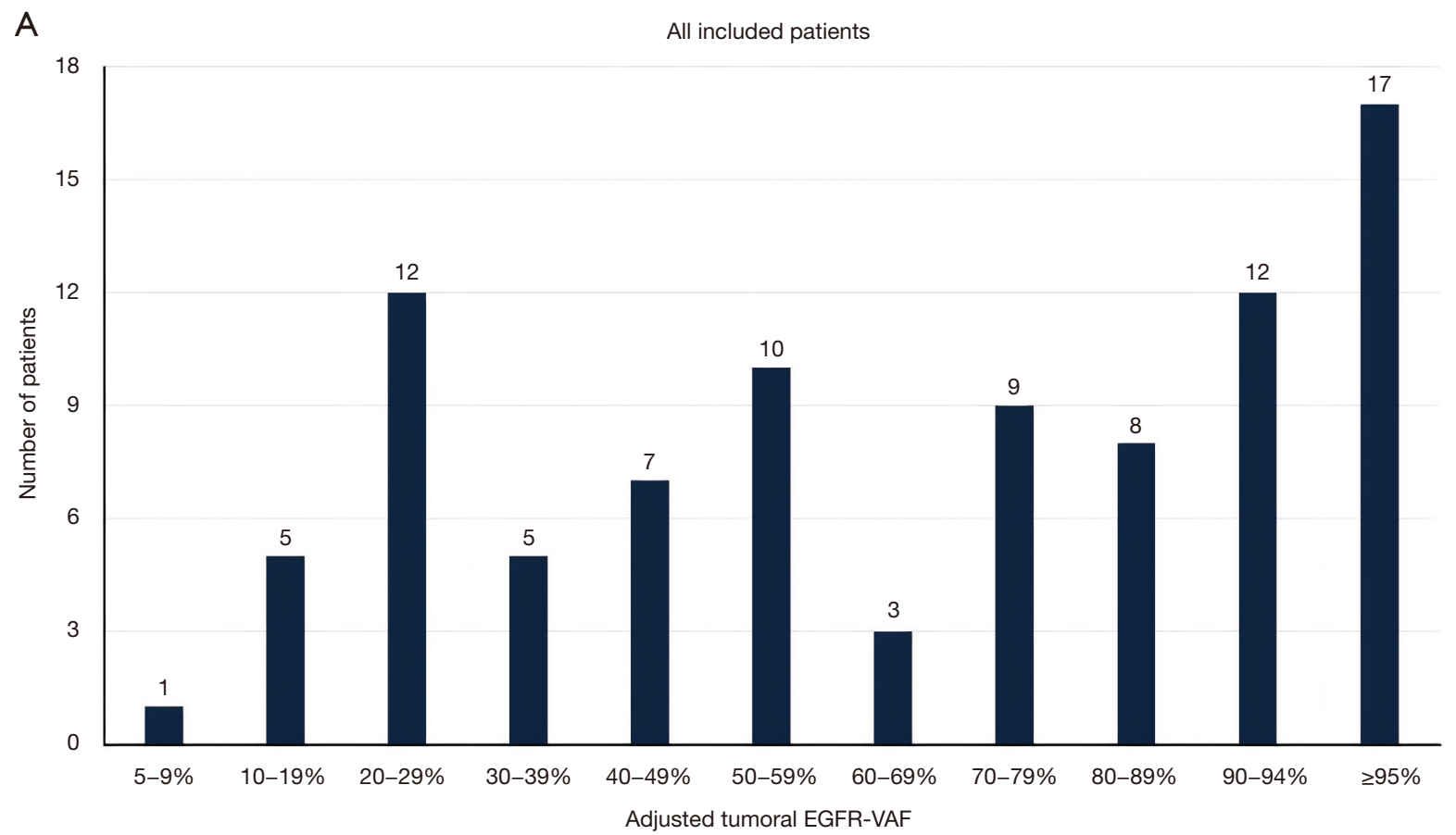

B

Patients with EGFR exon 19 and exon 21 mutations

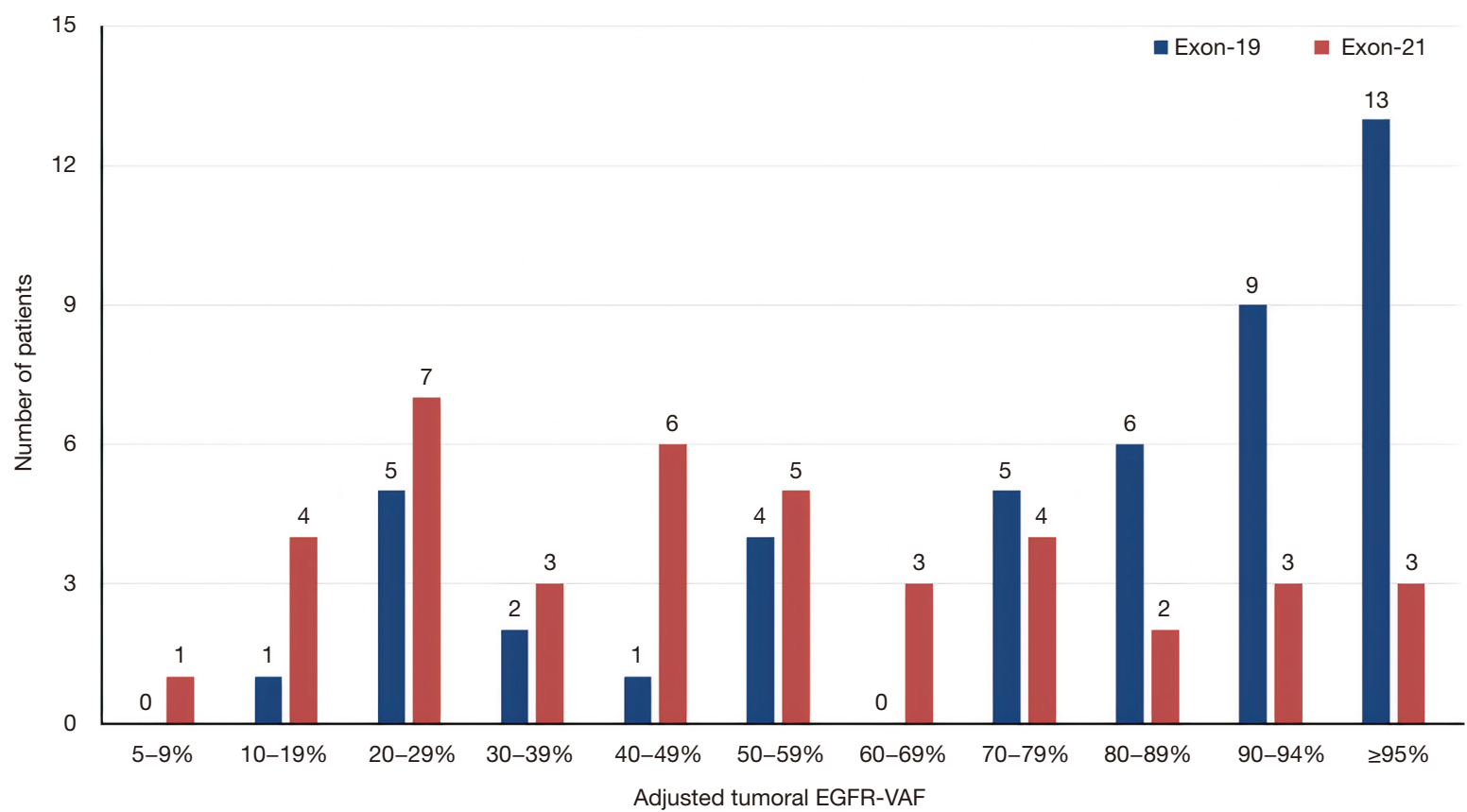

Figure 1 EGFR-aVAF of tumoral tissue in LADC patients. (A) Bar chart illustrating the distribution of all included LADC patients (n=89), according to tumoral EGFR-aVAF irrespective of specific exon mutations. (B) Distribution of LADC patients diagnosed with EGFR exon 19 and exon 21 mutations ( $\mathrm{n}=46$ and $\mathrm{n}=41$, respectively). EGFR, epidermal growth factor receptor; EGFR-aVAF, adjusted EGFR variant allele frequency; LADC, lung adenocarcinoma. 

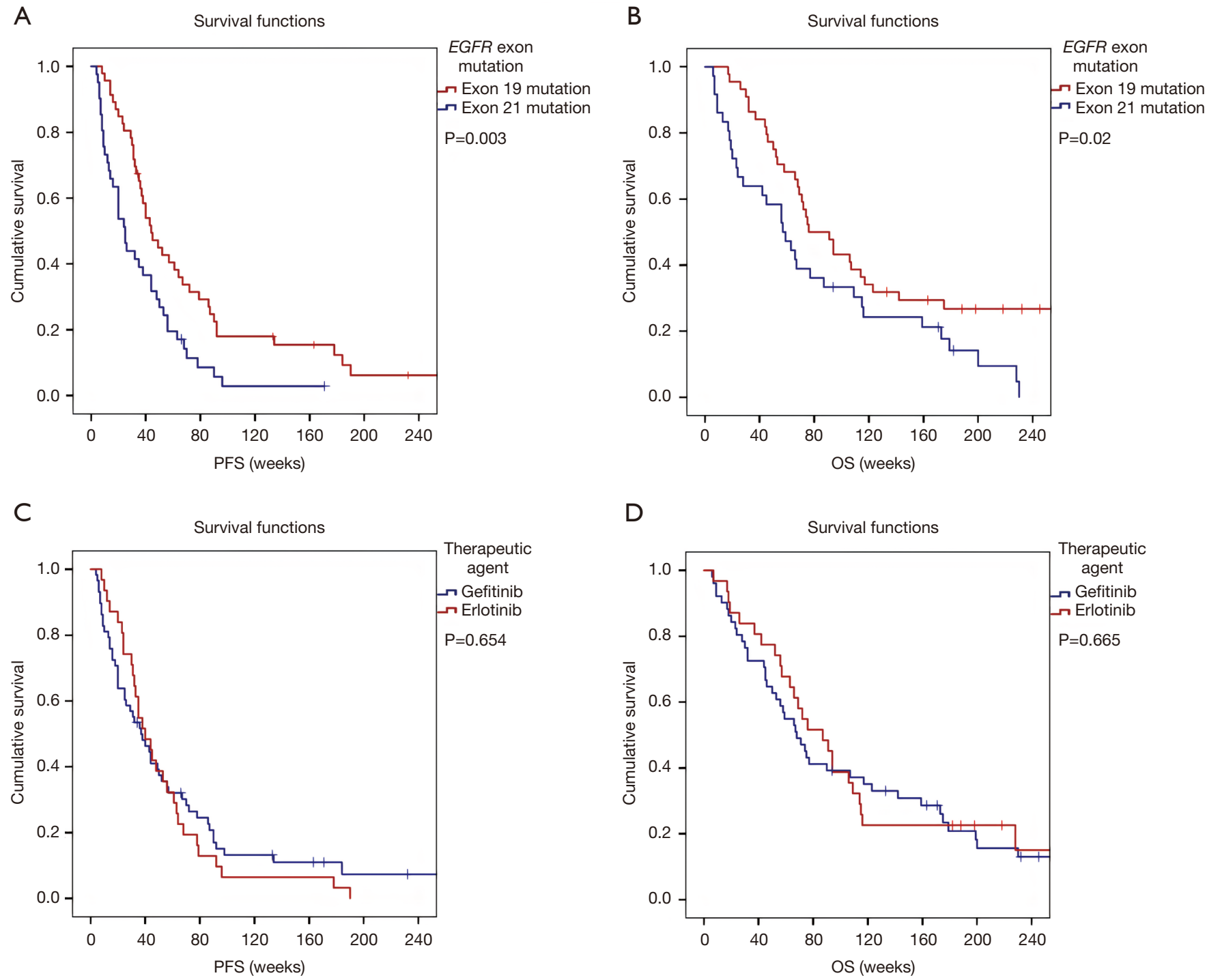

Figure 2 Kaplan-Meier plots for PFS and OS in patients with LADC according to specific EGFR exon mutations and therapeutic approaches. (A) LADC patients with tumors harboring EGFR exon 21 mutations had significantly shorter median PFS than those with exon 19 mutations (median PFSs were 25 vs. 44 weeks, respectively; $\mathrm{P}=0.003, \log$-rank test). (B) $E G F R$ exon 21 mutation was also associated with significantly shorter OS in these patients (vs. EGFR exon 19 mutations, median OSs were $57 v s .76$ weeks, respectively; $\mathrm{P}=0.02$, log-rank test). (C) No significant differences in PFS have been observed in patients treated with gefitinib vs. erlotinib (median PFSs were 37 vs. 40 weeks, respectively; $\mathrm{P}=0.654$, log-rank test). (D) Similarly, the OS also did not differ significantly between the patients treated with gefitinib $v s$. erlotinib (median OSs were 68 vs. 87 weeks, respectively; $\mathrm{P}=0.665$, log-rank test). PFS, progression-free survival; OS, overall survival; LADC, lung adenocarcinoma; EGFR, epidermal growth factor receptor.

relevant molecular pathological characteristics is crucial $(28,29)$. Targeting EGFR is a promising strategy for treating LADC patients, since numerous studies over the past decade have shown that the TKI inhibitors gefitinib and erlotinib are effective for advanced-stage NSCLCs harboring EGFR sensitizing mutations (30,31). Still, the efficacy of TKIs is not consistent for every patient and not all patients with $E G F R$-activating mutation show similar response rates and PFSs (18). Hence, there is an urgent need for identifying valid predictive and prognostic factors that enable clinicians to effectively select the patients who may benefit more from EGFR-TKI treatment. Early 


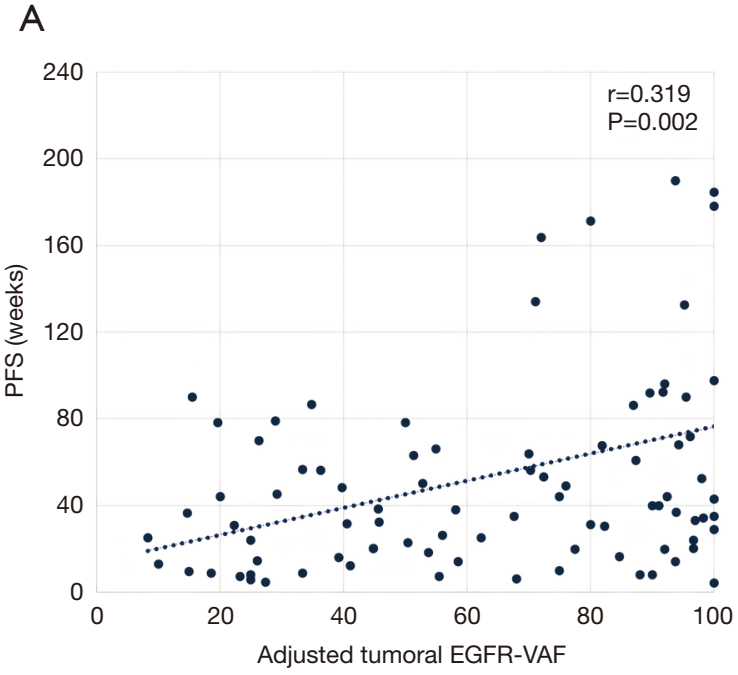

C

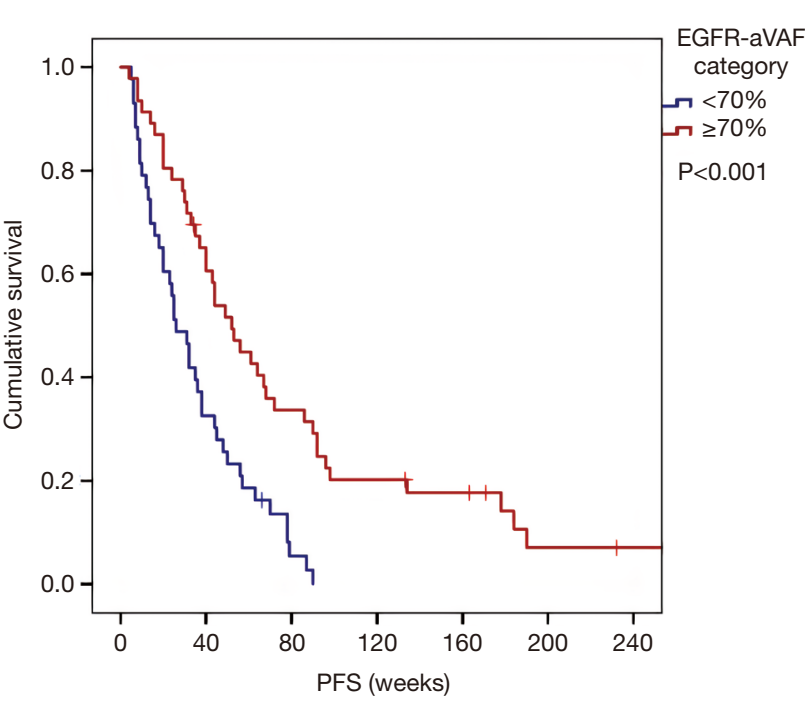

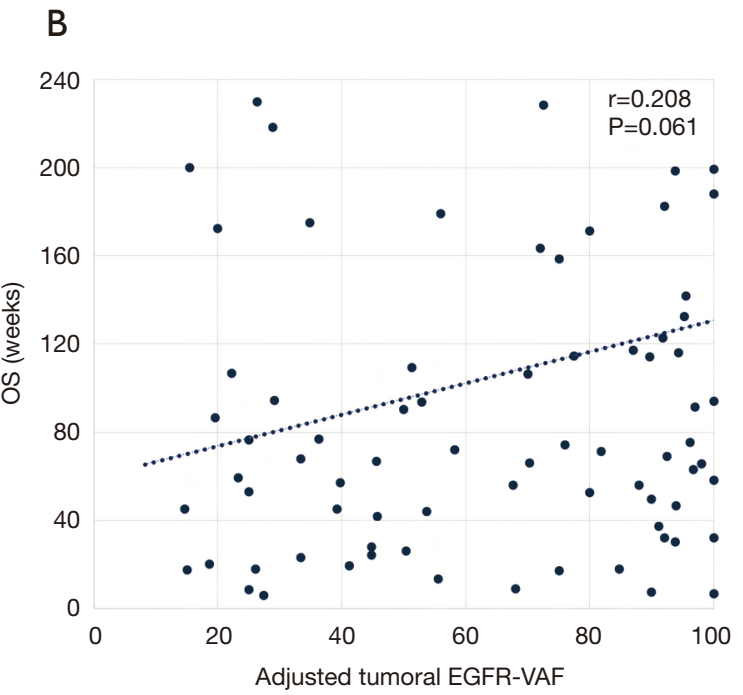

D Survival functions

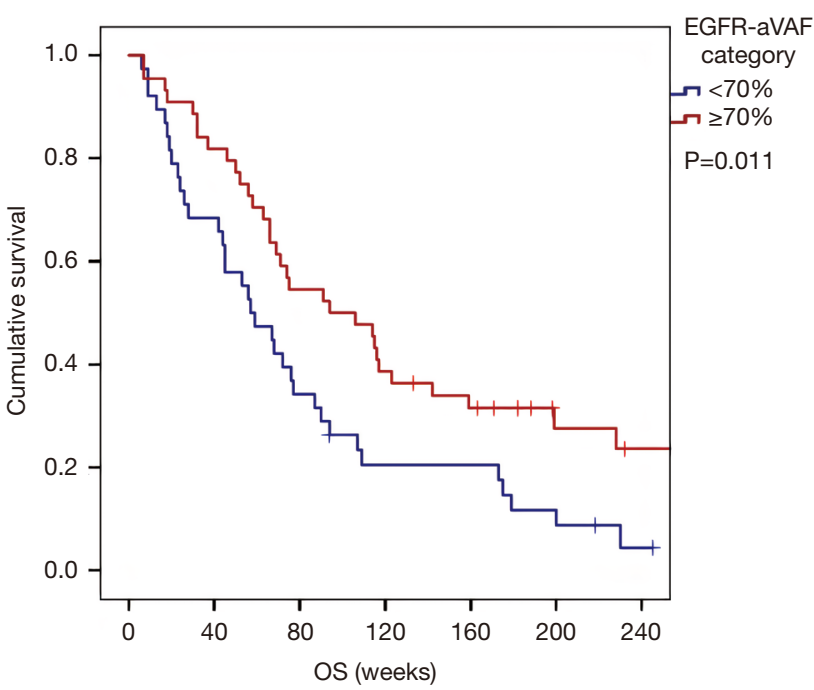

Figure 3 Scatter plots and Kaplan-Meier estimates for PFS and OS in LADC patients according to EGFR-aVAF. (A) Scatter plot showing significant positive linear correlation between tumoral EGFR-aVAF and PFS ( $r=0.319 ; \mathrm{P}=0.002$, Spearman's correlation) (each dot represents a single patient, and the dashed line shows the linear trendline). (B) Statistically non-significant, although clinically notable correlation was found between EGFR-VAF and OS ( $\mathrm{r}=0.208$; P=0.061, Spearman's correlation). (C) Patients with tumoral EGFR-aVAF $\geq 70 \%$ had significantly longer PFS than those in the EGFR-aVAF low ( $<70 \%$ ) group (median PFSs were 52 vs. 26 weeks, respectively; P<0.001, logrank test). (D) Similarly, the median OS was also significantly increased in patients with high ( $\geq 70 \%)$ EGFR-aVAF [vs. those with low (<70\%) EGFR-aVAF, median OSs were 94 vs. 57 weeks, respectively; P=0.011, log-rank test]. PFS, progression-free survival; OS, overall survival; LADC, lung adenocarcinoma; EGFR, epidermal growth factor receptor; EGFR-aVAF, adjusted EGFR variant allele frequency.

in 2011, Zhou et al. reported that the relative EGFR mutational abundance might predict the therapy response to gefitinib in advanced-stage Asian NSCLC patients, yet the predictive value and clinicopathological significance of
EGFR-aVAF is still controversial, especially in Caucasian patients (19). Therefore, the aim of this study was to assess the clinicopathological significance of EGFR-aVAF and to evaluate its predictive and prognostic relevance in a 
Table 2 Multivariate Cox regression model for clinicopathological variables influencing the PFS

\begin{tabular}{|c|c|}
\hline Clinicopathological parameters & PFS \\
\hline \multicolumn{2}{|l|}{ EGFR-aVAF (continuous) } \\
\hline HR & -0.009 \\
\hline $95 \% \mathrm{Cl}$ & $(0.982-0.999)$ \\
\hline $\mathrm{P}$ & 0.042 \\
\hline \multicolumn{2}{|c|}{ EGFR exon mutation (exon 19 vs. exon 21) } \\
\hline $\mathrm{HR}$ & 0.284 \\
\hline $95 \% \mathrm{Cl}$ & $(1.017-1.735)$ \\
\hline $\mathrm{P}$ & 0.037 \\
\hline \multicolumn{2}{|l|}{ Age (continuous) } \\
\hline $\mathrm{HR}$ & -0.021 \\
\hline $95 \% \mathrm{Cl}$ & $(0.958-1.001)$ \\
\hline $\mathrm{P}$ & 0.06 \\
\hline \multicolumn{2}{|l|}{ Gender (male vs. female) } \\
\hline HR & 0.460 \\
\hline $95 \% \mathrm{Cl}$ & $(0.913-2.747)$ \\
\hline$P$ & 0.102 \\
\hline \multicolumn{2}{|c|}{ Therapeutic agent (gefitinib vs. erlotinib) } \\
\hline HR & -0.032 \\
\hline $95 \% \mathrm{Cl}$ & $(0.595-1.579)$ \\
\hline$P$ & 0.899 \\
\hline \multicolumn{2}{|c|}{ Treatment line (first- vs. second-line) } \\
\hline HR & -0.013 \\
\hline $95 \% \mathrm{Cl}$ & $(0.607-1.603)$ \\
\hline$P$ & 0.957 \\
\hline
\end{tabular}

PFS, progression-free survival; EGFR, epidermal growth factor receptor; EGFR-aVAF, adjusted EGFR variant allele frequency; $\mathrm{HR}$, hazard ratio; $\mathrm{Cl}$, confidence interval.

homogenous cohort of Hungarian LADC patients treated with EGFR-TKIs.

First, we analyzed the association of major clinicopathological characteristics and tumoral EGFRaVAF. Our results revealed that a considerable proportion of LADCs contain a heterogeneous population of both EGFR mutated and non-mutated cancer cells since the majority of all included cases showed an EGFR-aVAF between $5 \%$ and $94 \%$ and only 17 patients exhibited EGFR-aVAF $\geq 95 \%$. This finding is in line with previously published data also suggesting that only a certain percentage of TCs carry heterozygous activating mutations in NSCLC patients, while other TCs carry wild-type $\operatorname{EGFR}(21,22)$. Accordingly, this might explain the controversial response rates seen in EGFR-TKI-treated patients. In the current study, $2.2 \%$ of patients carried exon 18 EGFR mutations, therefore the incidence rate is similar to other studies. However, due to the small number of patients harboring exon 18 mutations, subgroup specific statistical calculations were performed without these patients (15). Importantly, we found that the aVAF of the tumoral tissue was significantly higher in patients harboring EGFR exon 19 mutations than those with exon 21 mutated tumors. This ratio is in line with a previously published Asian study, however, to the best of our knowledge, ours is the first detailed evaluation of tumoral EGFR-aVAF with regards to specific EGFR exon mutations in Caucasian patients (23).

Next, in order to assess the clinical relevance of this heterogeneity in EGFR-aVAF between the patients harboring exon 19 vs. exon 21 mutations, we investigated the prognostic and predictive relevance of the aforementioned EGFR exon alterations. As expected, patients harboring EGFR exon 19 mutations indeed had significantly longer PFS than those with EGFR exon 21 mutations. These findings are in line with previously published data also suggesting a significant advantage in PFS for patients carrying exon 19 deletions in comparison with those carrying EGFR exon 21 mutations (32-35). In addition, based on a recent study on 55 metastatic NSCLC patients, exon 19-mutated patients tend to have better survival outcomes than patients with exon 18 pointmutations as well (15). To date, the mechanism underlying the different sensitivities to EGFR-TKI treatment between exon 19 and exon 21 mutated tumors remains to be elucidated (34). Based on our results a possible explanation might be that EGFR-aVAF of tumoral tissue is significantly higher in EGFR exon 19 mutated patients compared to patients harboring exon 21 mutations and thus EGFRTKIs might be more effective in these patients. Meanwhile, others suggest that the better survival outcomes with EGFR exon 19 than exon 21 mutations might be due to differential inhibition of downstream signals, since EGFRTKIs inhibit the phosphorylation of EGFR, Akt, and Erk to a greater degree in exon 19 deletion cells than in exon 21 mutated cells (36). Furthermore, an additional explanation might be that exon 19 deletions and 21 mutations present different intrinsic sensitivities to the EGFR-TKIs $(34,37)$. Importantly, different mutations in the same exon might 
also indicate different predictive roles since non-L747 to E749 (LRE) deletions has a worse response to TKIs than LRE deletions but we had no data on the type of deletions in exon 19 (38). Altogether, the biology that lies behind the responsiveness to EGFR-TKIs with regards to EGFR mutational subtypes is yet to be elucidated, however, our findings might provide background for future studies. In line with the PFS data, EGFR exon 19 mutations were also associated with improved OS compared to exon 21 mutations. As for treatment-related data, no significant differences were observed in PFS or OS regarding treatment line and therapeutic agents, which is in line with the findings of others (39-42).

Finally, we investigated the predictive and prognostic relevance of tumoral EGFR-aVAF and a statistically significant moderate positive linear correlation was found between EGFR-aVAF and PFS. Notably, we also found that high $(\geq 70 \%)$ tumoral EGFR-aVAF was associated both with improved median PFS and OS, with a clinically relevant difference between low and high subgroups of 26 and 37 weeks, respectively. It should be noted, however, that the patients were divided into low and high EGFRaVAF subgroups based on the median value in our dataset, therefore, until further validation, caution is needed when using it as a cut-off value in future studies. Our results are of high clinical importance because previous studies have only focused on whether the mutation is positive, and only a few investigated the predictive role of the relative EGFR mutational abundance $(19,23,24)$. Yet, to our knowledge, our study is the first investigating the predictive and prognostic relevance of the exact value of EGFR-aVAF in Caucasian patients and, moreover, the first suggesting a clinically relevant threshold for predicting treatment response in these patients. In support of this, multivariate Cox regression analysis also revealed that EGFR-aVAF at diagnosis influenced PFS independently from age, gender, therapeutic agent, treatment line, and type of EGFR exon mutation. These results might partly explain why the efficacy of TKIs is not consistent for every patient harboring a certain type of EGFR mutation. Accordingly, quantitative diagnosis methods of EGFR-aVAF may help to select patients who are most or least likely to benefit from EGFR-TKIs. Importantly, however, current clinical treatment protocols with regards to EFGR-TKI are still primarily based on the absence or presence of activating EGFR mutations (25). Accordingly, until future validation, the clinicians should choose the most appropriate treatment for their patients regardless of EGFR-aVAF status.
Nevertheless, changes in EGFR-aVAF might also occur during cancer progression and therapy. For instance, a recent study suggests that cancer genome in colorectal cancer patients adapts dynamically to pulsatile drug schedules and the abundance of resistance mutations could increase after long-time targeted therapies (43). Therefore, dynamic monitoring of EGFR-aVAF during therapy is also warranted.

There are several limitations in our study. Despite the fact that our cohort was homogenous the final number of patients harboring $E G F R$ mutations was relatively small due to our strict inclusion/exclusion criteria. Nevertheless, our cohort provided the opportunity to draw some conclusions that evidently need to be validated in additional studies. Another limitation of our study is its retrospective nature with given limitations in interpreting the results. Thus, some of our results need to be confirmed in a prospective setting. Loss of heterozygosity and EGFR amplification occurs frequently in LADC patients harboring $E G F R$ activating mutations and could serve as an indicator for better response from EGFR-TKI treatment (44-46). Accordingly, both of the aforementioned genetic alterations might also correlate with higher aVAF values, yet we did not investigate the presence of these alterations since they are not part of the routine mutational analyses in Hungary. Finally, all included patients were treated with firstgeneration EGFR-TKI erlotinib and gefitinib, yet these inhibitors are being slowly replaced by second- and thirdgeneration EGFR-TKIs in the clinical practice. All in all, taken into account all the aforementioned potential study limitations, caution is needed when interpreting the results of the present study and further analyses are warranted to clarify the exact predictive role of EGFR-aVAF in EGFRTKI-treated LADC patients.

\section{Conclusions}

To conclude, our study suggests that EGFR-aVAF of tumoral tissue predicts the extent of benefit from EGFRTKI treatment. Moreover, in regards with exon specific mutations, the average EGFR-aVAF is higher among patients with exon 19 deletions thus confirming the longer PFS and OS of these patients. Our results might as well explain why the duration of response of some EGFR mutant patients was not as long as expected when no resistance related abnormality was found. Altogether, by shedding light on the predictive and prognostic relevance of EGFRaVAF, our results might help to improve patient selection 
and treatment in advanced-stage LADC patients harboring EGFR-sensitizing mutations.

\section{Acknowledgments}

The authors are grateful to Laszlo Gobel for his technical help in the development of the clinical database. The authors would also like to thank the following clinicians for providing information concerning the clinicopathological data of included patients: Zsuzsanna Szalai, Gabriella Meszegeto and Istvan Albert.

Funding: BD, JM and CB acknowledge funding from the Hungarian National Research, Development and Innovation Office (KNN121510, KH130356, BD; NAP22017-1.2.1- NKP-0002, K129065, JM; NVKP_16-1-20160004, CB). BD and VL were supported by the Austrian Science Fund (FWF I3522, VL; FWF I3977 and I4677, $\mathrm{BD})$. VL is a recipient of the Bolyai Research Scholarship of the Hungarian Academy of Sciences and the UNKP19-4 New National Excellence Program of the Ministry for Innovation and Technology. ZM was supported by the UNKP-20-3 New National Excellence Program of the Ministry for Innovation and Technology. CB was supported by the Higher Education Institutional Excellence Programme of the Ministry of Human Capacities in Hungary within the framework of the Molecular Biology Thematic Programme of the Semmelweis University.

\section{Footnote}

Reporting Checklist: The authors have completed the STROBE reporting checklist. Available at http://dx.doi. org/10.21037/tlcr-20-814

Peer Review File: Available at http://dx.doi.org/10.21037/ tlcr-20-814

Conflicts of Interest: All authors have completed the ICMJE uniform disclosure form (available at http://dx.doi. org/10.21037/tlcr-20-814). The authors have no conflicts of interest to declare.

Ethical Statement: The authors are accountable for all aspects of the work in ensuring that questions related to the accuracy or integrity of any part of the work are appropriately investigated and resolved. The present study was directed in accordance with the guidelines of the Helsinki Declaration (revised in 2013) of the World
Medical Association. The study was approved by the national level ethics committee (Hungarian Scientific and Research Ethics Committee of the Medical Research Council, ETT-TUKEB, 7214-1/2016/EKU). The need for individual informed consent for this retrospective study was waived. After clinical information was collected, patient identifiers were removed, and subsequently, patients cannot be identified either directly or indirectly.

Open Access Statement: This is an Open Access article distributed in accordance with the Creative Commons Attribution-NonCommercial-NoDerivs 4.0 International License (CC BY-NC-ND 4.0), which permits the noncommercial replication and distribution of the article with the strict proviso that no changes or edits are made and the original work is properly cited (including links to both the formal publication through the relevant DOI and the license). See: https://creativecommons.org/licenses/by-nc-nd/4.0/.

\section{References}

1. Bray F, Ferlay J, Soerjomataram I, et al. Global cancer statistics 2018: GLOBOCAN estimates of incidence and mortality worldwide for 36 cancers in 185 countries. CA Cancer J Clin 2018;68:394-424.

2. Revannasiddaiah S, Thakur P, Bhardwaj B, et al. Pulmonary adenocarcinoma: implications of the recent advances in molecular biology, treatment and the IASLC/ ATS/ERS classification. J Thorac Dis 2014;6:S502-25.

3. Midha A, Dearden S, McCormack R. EGFR mutation incidence in non-small-cell lung cancer of adenocarcinoma histology: a systematic review and global map by ethnicity (mutMapII). Am J Cancer Res 2015;5:2892-911.

4. Harrison PT, Vyse S, Huang PH. Rare epidermal growth factor receptor (EGFR) mutations in non-small cell lung cancer. Semin Cancer Biol 2020;61:167-79.

5. Bethune G, Bethune D, Ridgway N, et al. Epidermal growth factor receptor (EGFR) in lung cancer: an overview and update. J Thorac Dis 2010;2:48-51.

6. da Cunha Santos G, Shepherd FA, Tsao MS. EGFR mutations and lung cancer. Annu Rev Pathol 2011;6:49-69.

7. Inamura $K$, Ninomiya $H$, Ishikawa $Y$, et al. Is the epidermal growth factor receptor status in lung cancers reflected in clinicopathologic features? Arch Pathol Lab Med 2010;134:66-72.

8. Sigismund S, Avanzato D, Lanzetti L. Emerging functions of the EGFR in cancer. Mol Oncol 2018;12:3-20.

9. Kosaka T, Yatabe Y, Endoh H, et al. Mutations of 
the epidermal growth factor receptor gene in lung cancer: biological and clinical implications. Cancer Res 2004;64:8919-23.

10. Beau-Faller M, Prim N, Ruppert AM, et al. Rare EGFR exon 18 and exon 20 mutations in non-small-cell lung cancer on 10117 patients: a multicentre observational study by the French ERMETIC-IFCT network. Ann Oncol 2014;25:126-31.

11. Kobayashi S, Boggon TJ, Dayaram T, et al. EGFR mutation and resistance of non-small-cell lung cancer to gefitinib. N Engl J Med 2005;352:786-92.

12. Yu HA, Riely GJ. Second-generation epidermal growth factor receptor tyrosine kinase inhibitors in lung cancers. J Natl Compr Canc Netw 2013;11:161-9.

13. Andrews Wright NM, Goss GD. Third-generation epidermal growth factor receptor tyrosine kinase inhibitors for the treatment of non-small cell lung cancer. Transl Lung Cancer Res 2019;8:S247-64.

14. Maemondo M, Inoue A, Kobayashi K, et al. Gefitinib or chemotherapy for non-small-cell lung cancer with mutated EGFR. N Engl J Med 2010;362:2380-8.

15. Rossi S, D'Argento E, Basso M, et al. Different EGFR gene mutations in exon 18,19 and 21 as prognostic and predictive markers in NSCLC: a single institution analysis. Mol Diagn Ther 2016;20:55-63.

16. Tamura K, Okamoto I, Kashii T, et al. Multicentre prospective phase II trial of gefitinib for advanced nonsmall cell lung cancer with epidermal growth factor receptor mutations: results of the West Japan Thoracic Oncology Group trial (WJTOG0403). Br J Cancer 2008;98:907-14.

17. Kim SY, Myung JK, Kim HR, et al. Factors that predict clinical benefit of EGFR TKI therapy in patients with EGFR wild-type lung adenocarcinoma. Tuberc Respir Dis (Seoul) 2019;82:62-70.

18. Zhang X, Chang A. Molecular predictors of EGFR-TKI sensitivity in advanced non-small cell lung cancer. Int J Med Sci 2008;5:209-17.

19. Zhou Q, Zhang XC, Chen ZH, et al. Relative abundance of EGFR mutations predicts benefit from gefitinib treatment for advanced non-small-cell lung cancer. J Clin Oncol 2011;29:3316-21.

20. Zhu Y, Guo Z, Liu Y, et al. A novel ARMS-based assay for the quantification of EGFR mutations in patients with lung adenocarcinoma. Oncol Lett 2018;15:2905-12.

21. Taniguchi K, Okami J, Kodama K, et al. Intratumor heterogeneity of epidermal growth factor receptor mutations in lung cancer and its correlation to the response to gefitinib. Cancer Sci 2008;99:929-35.

22. Jiang SX, Yamashita K, Yamamoto M, et al. EGFR genetic heterogeneity of nonsmall cell lung cancers contributing to acquired gefitinib resistance. Int J Cancer 2008;123:2480-6.

23. Li X, Cai W, Yang G, et al. Comprehensive analysis of EGFR-mutant abundance and its effect on efficacy of EGFR TKIs in advanced NSCLC with EGFR mutations. J Thorac Oncol 2017;12:1388-97.

24. Wang H, Zhang M, Tang W, et al. Mutation abundance affects the therapeutic efficacy of EGFR-TKI in patients with advanced lung adenocarcinoma: a retrospective analysis. Cancer Biol Ther 2018;19:687-94.

25. Ettinger DS, Wood DE, Aggarwal C, et al. NCCN guidelines insights: non-small cell lung cancer, version 1.2020. J Natl Compr Canc Netw 2019;17:1464-72.

26. Schwartz LH, Litiere S, de Vries E, et al. RECIST 1.1-update and clarification: from the RECIST committee. Eur J Cancer 2016;62:132-7.

27. Ratner B. The correlation coefficient: Its values range between $+1 /-1$, or do they? J Target Meas Anal Mark 2009;17:139-42.

28. Lohinai Z, Hoda MA, Fabian K, et al. Distinct epidemiology and clinical consequence of classic versus rare EGFR mutations in lung adenocarcinoma. J Thorac Oncol 2015;10:738-46.

29. Raparia K, Villa C, DeCamp MM, et al. Molecular profiling in non-small cell lung cancer: a step toward personalized medicine. Arch Pathol Lab Med 2013;137:481-91.

30. Thatcher N, Chang A, Parikh P, et al. Gefitinib plus best supportive care in previously treated patients with refractory advanced non-small-cell lung cancer: results from a randomised, placebo-controlled, multicentre study (Iressa Survival Evaluation in Lung Cancer). Lancet 2005;366:1527-37.

31. Shepherd FA, Rodrigues Pereira J, Ciuleanu T, et al. Erlotinib in previously treated non-small-cell lung cancer. N Engl J Med 2005;353:123-32.

32. Jackman DM, Yeap BY, Sequist LV, et al. Exon 19 deletion mutations of epidermal growth factor receptor are associated with prolonged survival in non-small cell lung cancer patients treated with gefitinib or erlotinib. Clin Cancer Res 2006;12:3908-14.

33. Riely GJ, Pao W, Pham D, et al. Clinical course of patients with non-small cell lung cancer and epidermal growth factor receptor exon 19 and exon 21 mutations treated with gefitinib or erlotinib. Clin Cancer Res 2006;12:839-44.

34. Hong $\mathrm{W}, \mathrm{Wu} \mathrm{Q}$, Zhang J, et al. Prognostic value of EGFR 
19-del and 21-L858R mutations in patients with non-small cell lung cancer. Oncol Lett 2019;18:3887-95.

35. Jiang H, Zhu M, Li Y, et al. Association between EGFR exon 19 or exon 21 mutations and survival rates after firstline EGFR-TKI treatment in patients with non-small cell lung cancer. Mol Clin Oncol 2019;11:301-8.

36. Zhu JQ, Zhong WZ, Zhang GC, et al. Better survival with EGFR exon 19 than exon 21 mutations in gefitinibtreated non-small cell lung cancer patients is due to differential inhibition of downstream signals. Cancer Lett 2008;265:307-17.

37. Paez JG, Janne PA, Lee JC, et al. EGFR mutations in lung cancer: correlation with clinical response to gefitinib therapy. Science 2004;304:1497-500.

38. Chung KP, Wu SG, Wu JY, et al. Clinical outcomes in non-small cell lung cancers harboring different exon 19 deletions in EGFR. Clin Cancer Res 2012;18:3470-7.

39. Zhang W, Wei Y, Yu D, et al. Gefitinib provides similar effectiveness and improved safety than erlotinib for advanced non-small cell lung cancer: a meta-analysis. Medicine (Baltimore) 2018;97:e0460.

40. Chai CS, Liam CK, Pang YK, et al. Gefitinib versus erlotinib as first-line treatment in EGFR mutant advanced lung adenocarcinoma. Eur Respir J 2016;48:OA3341.

Cite this article as: Gieszer B, Megyesfalvi Z, Dulai V, Papay J, Kovalszky I, Timar J, Fillinger J, Harko T, Pipek O, Teglasi V, Regos E, Papp G, Szallasi Z, Laszlo V, Renyi-Vamos F, Galffy G, Bodor C, Dome B, Moldvay J. EGFR variant allele frequency predicts EGFR-TKI efficacy in lung adenocarcinoma: a multicenter study. Transl Lung Cancer Res 2021;10(2):662-674. doi: $10.21037 /$ tlcr-20-814
41. Wu JY, Yu CJ, Yang CH, et al. First- or second-line therapy with gefitinib produces equal survival in nonsmall cell lung cancer. Am J Respir Crit Care Med 2008;178:847-53.

42. Massuti B, Morán T, Porta R, et al. Multicenter prospective trial of customized erlotinib for advanced non-small cell lung cancer (NSCLC) patients (p) with epidermal growth factor receptor (EGFR) mutations: Final results of the Spanish Lung Cancer Group (SLCG) trial. J Clin Oncol 2009;27:8023.

43. Siravegna G, Mussolin B, Buscarino M, et al. Clonal evolution and resistance to EGFR blockade in the blood of colorectal cancer patients. Nat Med 2015;21:795-801.

44. Ma ES, Wong CL, Siu D, et al. Amplification, mutation and loss of heterozygosity of the EGFR gene in metastatic lung cancer. Int J Cancer 2007;120:1828-31; author reply 1832-3.

45. Shan L, Wang Z, Guo L, et al. Concurrence of EGFR amplification and sensitizing mutations indicate a better survival benefit from EGFR-TKI therapy in lung adenocarcinoma patients. Lung Cancer 2015;89:337-42.

46. Hayes DN, McLeod HL. EGFR regulation by microRNA in lung cancer: a rose by any other name ... is an increasingly complicated rose. Ann Oncol 2008;19:1036-7. 
Supplementary

Table S1 List of participating medical centers

Medical center Number of patients

Torokbalint County Institute of Pulmonology, Torokbalint, Hungary

Department of Pulmonology of the Semmelweis University, Budapest, Hungary

Veszprem County Lung Hospital, Farkasgyepu, Hungary

9

Uzsoki Hospital, Budapest, Hungary

National Koranyi Institute of Pulmonology, Budapest, Hungary

County Hospital Fejer, Szent Gyorgy Hospital, Szekesfehervar, Hungary

County Hospital Zala, Zalaegerszeg, Hungary

County Hospital Gyor, Petz Aladar Hospital, Gyor, Hungary

$3^{\text {rd }}$ Department of Internal Medicine of the Semmelweis University, Budapest, Hungary 

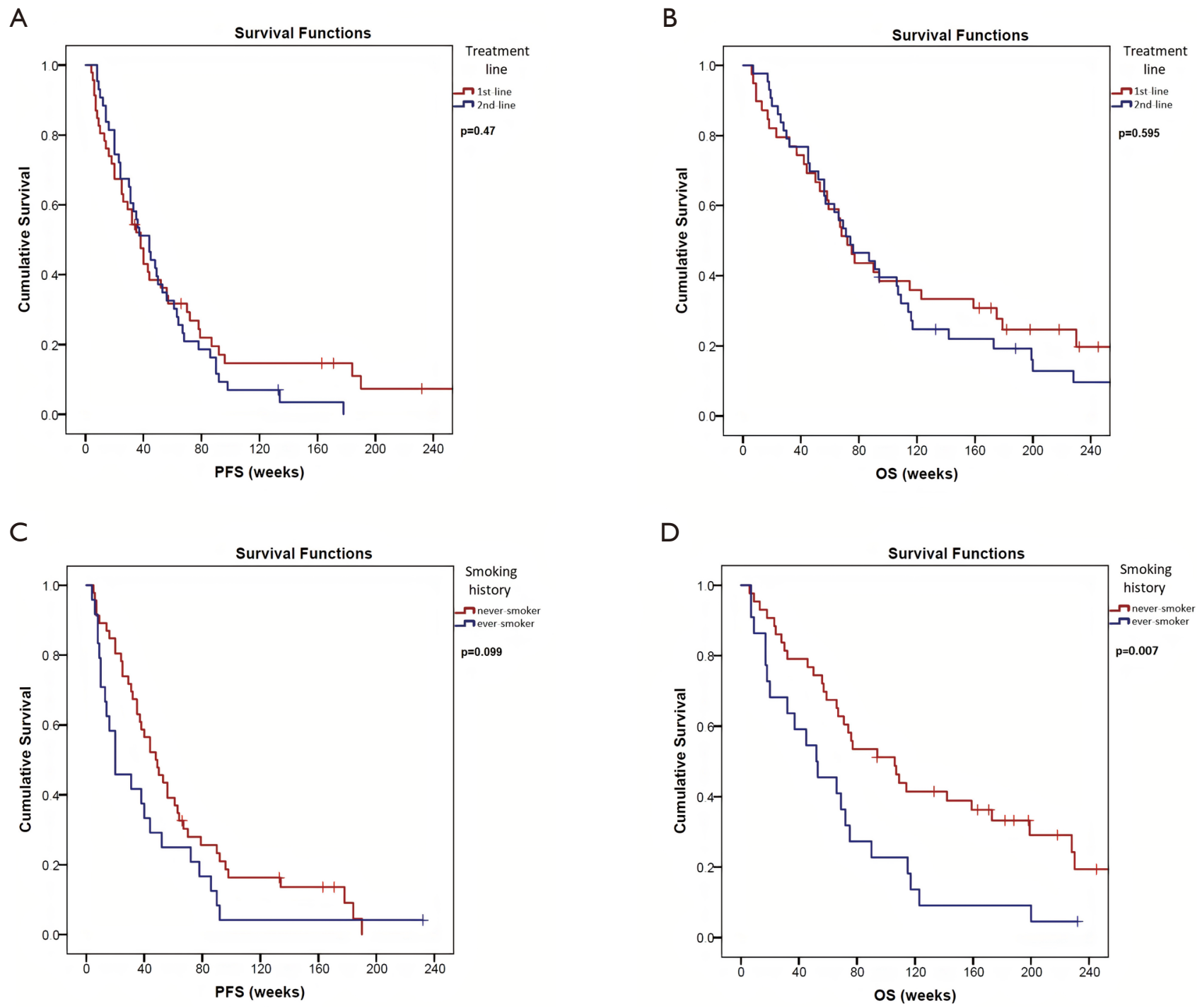

Figure S1 Comparison of survival outcomes in patients with advanced LADC with regards to treatment line and smoking status. (A) No significant differences in PFS have been observed between patients receiving EGFR-TKI in first- vs. second-line (median PFSs were 38 vs. 44 weeks, respectively; $\mathrm{P}=0.47$, log-rank test). (B) Patients receiving EGFR-TKI in first-line had a similar OS compared to patients receiving EGFR-TKI in second-line (median OSs were 72 vs. 74 weeks, respectively; $\mathrm{P}=0.595$, log rank-test). (C) Statistically non-significant, although clinically notable difference was found in PFS between never-smoker and ever-smoker patients (median PFSs were 48 s. 20 weeks, respectively; $\mathrm{P}=0.099$, log-rank test). (D) Never-smoker patients had significantly improved OS (vs. ever-smokers; median OSs were 106 vs. 52 weeks, respectively; $\mathrm{P}=0.007$, log-rank test). LADC, lung adenocarcinoma; PFS, progression-free survival; EGFR, epidermal growth factor receptor; EGFR-TKI, EGFR tyrosine kinase inhibitor; OS, overall survival. 